\title{
Synchronization and Antisynchronization for a Class of Chaotic Systems by a Simple Adaptive Controller
}

\author{
Ling Ren and Rongwei Guo \\ School of Science, Qilu University of Technology, Jinan 250353, China \\ Correspondence should be addressed to Rongwei Guo; rongwei_guo@163.com
}

Received 21 August 2015; Accepted 20 October 2015

Academic Editor: Jonathan N. Blakely

Copyright ( 2015 L. Ren and R. Guo. This is an open access article distributed under the Creative Commons Attribution License, which permits unrestricted use, distribution, and reproduction in any medium, provided the original work is properly cited.

\begin{abstract}
This paper investigates the synchronization and antisynchronization for a class of chaotic system. Firstly, a necessary and sufficient condition is proposed to synchronize and antisynchronization simultaneously for the chaotic systems. Secondly, two methods are obtained to realize coexistence of synchronization and antisynchronization in the chaotic systems, and the corresponding adaptive controllers are also given. Finally, two numerical examples with simulation verify the correctness and effectiveness of the obtained results.
\end{abstract}

\section{Introduction}

Since Lorenz firstly found the classical chaotic attractor in 1963 [1], as a most fascinating phenomenon in nonlinear dynamical system, chaos has been intensively studied over the past few decades; see $[2,3]$ and the references therein. It is well known that Pecora and Carroll firstly investigated the synchronization problem of chaotic systems in 1990 [4], and Ott et al. firstly presented a method to control chaotic systems successfully in 1990 in [5]. From then on, chaos control and chaos synchronization have received a great deal of attention in the area of nonlinear control as the significance of these two problems in both academic research and practical applications, and many important results were obtained; please refer to [6-11].

Up to date, several types of typical synchronization have been identified such as complete synchronization (CS), phase synchronization (PS), lag synchronization (LS), generalized synchronization (GS), anti-phase synchronization (AS), and projective synchronization (PS), and a variety of works have been done about the above problems; see $[6,7,12-16]$ and the references therein. It is well known that the master system synchronizes the slave system which is equivalent to the error system that is asymptotically stable. That is to say, chaos synchronization is equivalent to the error system which is asymptotically stable. Similarly, the master system antisynchronizes the slave system which is equivalent to the sum system that is also asymptotically stable. From the view of control theory [17], in order to design a simple and physical controller, the following condition is necessary; that is, $e=0$ is an equilibrium point of the unforced nominal error system $\dot{e}=f(y)-f(x), \dot{x}=f(x)$, where $e=y-x$, and $E=0$ is also an equilibrium point of the unforced nominal sum system $\dot{E}=f(y)+f(x), \dot{x}=f(x)$, where $E=y+x$. Obviously, $e=0$, that is, $y=x$, is an equilibrium point of the error system $\dot{e}=f(y)-f(x)$. Whereas, $E=0$, that is, $y=-x$, is an equilibrium point of the error system $\dot{E}=f(y)+f(x)$ if and only if $f(-x)=-f(x)$. Thus, the antisynchronization problem is more complex than the synchronization problem. However, this necessary condition is not considered in the most of the existing works on antisynchronization of chaotic systems [14, 15]. Although the authors have solved the antisynchronization of chaotic systems successfully, the controllers that have been obtained are complex; that is, some terms in those controllers are needed to counteract the redundant terms which make $E=0$ not the equilibrium point of the sum system $\dot{E}=f(y)+$ $f(x)$. For example, $x_{2} z_{2}+x_{1} z_{1}$ in $u_{2}$ of (14) counteracts the redundant term $-x_{2} z_{2}-x_{1} z_{1}$ in error system (13), and $-x_{1} y_{1}-x_{2} y_{2}$ in $u_{3}$ of (14) also does; for details please see [15].

It should be pointed out that most of the existing works focus on investigating the same kind synchronization in a given chaotic system; that is, all the states of the slave system have the same kind synchronization to the corresponding 
states of the master system. For example, when we say that two systems are synchronized (or antisynchronized, or lagsynchronized, or something else) with each other, it means that each pair of the states between the interactive systems is complete synchronous (or antisynchronous, or something else). In [18], the authors firstly pointed out the coexistence and switching of anticipating synchronization and lag synchronization in an optical system. From then on, some important results have been obtained; see [19, 20]. However, there are no results which can give some conditions or algorithms to select what variables in the master chaotic system which can synchronize or antisynchronize the corresponding variables in the slave chaotic systems have been published so far. Therefore, the coexistence of synchronization and antisynchronization of a class of chaotic or hyperchaotic systems needs further research.

Motivated by the above two reasons, we investigate the synchronization and antisynchronization for a class of chaotic systems in this paper. Firstly, for a class of chaotic systems, we obtain a necessary and sufficient condition with which the master system can synchronize and antisynchronize the slave system simultaneously. Secondly, we give two methods to realize coexistence of synchronization and antisynchronization in the chaotic systems and design the corresponding adaptive controllers. Finally, two numerical examples with simulation verify the correctness and effectiveness of the obtained results.

\section{Preliminary Knowledge}

This paper studies the synchronization and antisynchronization for a class of chaotic systems by adaptive control method. In order to develop this paper, some assumption and definitions are introduced firstly.

Assumption 1 (see [17]). $x_{e}=0$ is an equilibrium of the nonlinear system $\dot{x}=f(x)$; that is, $f\left(x_{e}\right)=0$.

Remark 2. Assumption 1 is a basic assumption of the system control theory. Without loss of generality, if $x_{e} \neq 0$, we can obtain a new system $\dot{y}=f\left(y+x_{e}\right)$ whose equilibrium is $y_{e}=$ 0 by making a coordinate transform $y=x-x_{e}$.

Definition 3 (see [12]). Consider the following chaotic system:

$$
\dot{x}=f(x),
$$

where $x \in \mathbb{R}^{n}$ is the state and $f(x)$ is a smooth nonlinear vector function.

Let system (1) be the master system; then the corresponding slave system with controller $u$ is given as

$$
\dot{y}=f(y)+u
$$

where $y \in \mathbb{R}^{n}$ is the state and $u=\left(u_{1}, u_{2}, \ldots, u_{n}\right)^{T}$ is the controller to be designed.

Let $e=y-x$, and the error system is described as

$$
\dot{e}=f(y)-f(x)+u=F(x, e)+u .
$$

We call master chaotic system (1) and slave system (2) reach complete synchronization if $\lim _{t \rightarrow \infty}\|e(t)\|=0$.

Definition 4 (see [14]). Consider master system (1) and slave system (2), let $E=y+x$, and the sum system is described as

$$
\dot{E}=f(y)+f(x)+u=G(x, E)+u .
$$

We say master system (1) and slave (2) get antisynchronization if $\lim _{t \rightarrow \infty}\|E(t)\|=0$.

Definition 5 (see [21]). Consider master system (1) and slave system (2). If error system (3) and sum system (4) can realize stabilization under the controllers $u=K(e)$ and $u=K(E)$, respectively, where $K($,$) is a smooth function, we say master$ system (1) synchronizes and antisynchronizes slave system (2) simultaneously; that is, slave system (1) can synchronize and antisynchronize slave system (2) using a controller with the same form.

Definition 6 (see [18]). Consider master system (1) and slave system (2). If there exists a controller $u$ satisfying $\lim _{t \rightarrow \infty} e_{i}=$ $\lim _{t \rightarrow \infty} x_{i}-y_{i}=0$ and $\lim _{t \rightarrow \infty} E_{j}=\lim _{t \rightarrow \infty} x_{j}+y_{j}=0$, where $i \neq j$, and $i, j \in \Lambda=\{1,2, \ldots, n\}$, we say master system (1) and slave system (2) can realize the coexistence of synchronization and antisynchronization. That is to say, some variables $\left(e_{i}\right)$ get synchronization, while some variables $\left(E_{j}\right)$ realize antisynchronization.

With the development of this paper, we introduce our previous result which can make the error system or the sum system reach stabilization.

Lemma 7 (see [13]). Consider error system (3). If $e_{i}=0$ and the remainder error system $\dot{e}_{k}=F_{k}\left(x, 0, e_{k}\right)$ is asymptotically stable, then the controlled error system is given as $\dot{e}_{i}=F_{i}(x, e)+$ $k_{1} e_{i}$, and $\dot{e}_{k}=F_{k}(x, e)$, where $i \neq k, i, k \in \Lambda$, and the feedback $k_{1}$ is updated according to the following update law:

$$
\dot{k}_{1}=-\gamma \sum_{i \in \Lambda} e_{i}^{2}
$$

Similarly, for sum system (4), if $E_{j}=0$ and the remainder sum system $\dot{E}_{l}=G_{l}\left(x, 0, E_{l}\right)$ is asymptotically stable, where $j \neq l, j, l \in \Lambda$, then the controlled sum system is given as $\dot{E}_{j}=G_{j}(x, E)+k_{1} E_{j}$, and $\dot{E}_{l}=G_{l}(x, E)$, and the feedback $k_{1}$ is updated according to the following update law:

$$
\dot{k}_{1}=-\gamma \sum_{j \in \Lambda} E_{j}^{2},
$$

where $\gamma$ is a positive constant.

\section{Main Results}

In this section, we firstly give a necessary and sufficient condition for a class of chaotic systems, by which we can determine whether master system (1) and slave system (2) realize synchronization and antisynchronization simultaneously or not. 
Theorem 8. Master system (1) and slave system (2) realize synchronization and antisynchronization simultaneously if and only if $f(-x)=-f(x)$; that is, $f(x)$ is the odd function.

$(\Rightarrow)$ Since $f(-x)=-f(x)$, obviously, $e=0$ and $E=0$ are the equilibria of unforced nominal error system (3) and sum system (4), that is, $u=0$, respectively. Then, if the controller $u=K(e)$ can stabilize error system (3), sum system (4) can be stabilized by the controller $u=$ $K(E)$.

$(\Leftarrow)$ If there exist the controllers $u=K(e)$ and $u=$ $K(E)$ stabilizing error systems (3) and (4), respectively, we can obtain thate $=0$ and $E=0$ are the equilibria of unforced nominal error system (3) and sum system (4), respectively. Then, $f(-x)=-f(x)$.

Remark 9. Although, the problem of synchronization and antisynchronization simultaneously of 4-dimension hyperchaotic system has been investigated in [22], no sufficient or necessary and sufficient condition for the general chaotic systems was proposed. Theorem 8 gives a necessary and sufficient condition for a class of chaotic systems.

If $f(x)$ is not an odd function, master system (1) and slave system (2) cannot realize synchronization and antisynchronization simultaneously according to Theorem 8 . Under this condition, they can reach the coexistence of synchronization and antisynchronization. Then, we give two methods to realize the coexistence of synchronization and antisynchronization for a class of chaotic systems. and let

Firstly, consider master system (1) and slave system (2),

$$
e=y-\alpha x,
$$

where $x, y$, and $e \in \mathbb{R}^{n}$ and $\alpha=\operatorname{Diag}\left(\alpha_{1}, \alpha_{2}, \alpha_{3}, \ldots, \alpha_{n}\right),\left|\alpha_{i}\right|=$ $1, i \in \Lambda$. The error system is given as

$$
\dot{e}=f(e+\alpha x)-f(x)+u .
$$

Remark 10. Obviously, if $\alpha_{i}=1, i \in \Lambda$, then master system (1) and slave system (2) reach complete synchronization. If some $\alpha_{i}=-1$, while some $\alpha_{j}=1, i \neq j \in \Lambda$, we say master system (1) and slave system (2) realize the coexistence of synchronization and antisynchronization.

According to Assumption 1, we give the following conclusion.

Theorem 11. Consider unforced nominal error system (8); that is, $u=0$. If $\alpha$ satisfies the condition $f(\alpha x)-f(x)=0$ and at least one $\alpha_{i}=-1$ and one $\alpha_{j}=1$, where $i, j \in \Lambda$, master system (1) and slave system (2) can reach coexistence of synchronization and antisynchronization.

Proof. According to Assumption 1, $e=0$ should be the equilibrium of unforced nominal error system (8), and thus $f(\alpha x)-f(x)=0$. And there exist at least one $\alpha_{i}=-1$ and one $\alpha_{j}=1$, where $i, j \in \Lambda$, and master system (1) and slave system (2) can reach coexistence of synchronization and antisynchronization according to Definition 6.

Remark 12. Theorem 11 gives a condition which can determine what variables in master system (1) can synchronize the corresponding variables in slave system (2), while other variables in master system (1) can antisynchronize the corresponding variables in slave system (2).

Inspired by the results in [16], we give another method to realize coexistence of synchronization and antisynchronization for a class of chaotic systems. First of all, master system (1) is rewritten as

$$
\dot{x}=\left(\begin{array}{c}
\dot{w} \\
\dot{z}
\end{array}\right)=f(x)=\left(\begin{array}{l}
M(z) w \\
N(w, z)
\end{array}\right),
$$

where $w=\left(x_{1}, x_{2}, \ldots, x_{r}\right)^{T}, z=\left(x_{r+1}, x_{r+2}, \ldots, x_{n}\right)$, if $N(-w, z)=N(w, z)$; that is, $N(w, z)$ is an even function, and we can get the following conclusion.

Theorem 13. Consider master system (1) and slave system (2), let $x=\left(w_{m}, z_{m}\right)^{T} \in \mathbb{R}^{n}, w_{m} \in \mathbb{R}^{r}, z_{m} \in \mathbb{R}^{n-r}$, and master system (1) can be written as

$$
\left(\begin{array}{c}
\dot{w}_{m} \\
\dot{z}_{m}
\end{array}\right)=\left(\begin{array}{c}
M\left(z_{m}\right) w_{m} \\
N\left(w_{m}, z_{m}\right)
\end{array}\right) .
$$

The slave system is described as

$$
\left(\begin{array}{c}
\dot{w}_{s} \\
\dot{z}_{s}
\end{array}\right)=\left(\begin{array}{c}
M\left(z_{s}\right) w_{s} \\
N\left(w_{s}, z_{s}\right)
\end{array}\right)+u,
$$

where $y=\left(w_{s}, z_{s}\right)^{T} \in \mathbb{R}^{n}, w_{s} \in \mathbb{R}^{r}, z_{s} \in \mathbb{R}^{n-r}, u=\left(u_{w}, u_{z}\right)^{T}$ is the controller to be designed. If $N(-w, z)=N(w, z)$, master system (1) and slave system (2) realize the coexistence of synchronization and antisynchronization.

Proof. Let $E=w_{m}+w_{s}, e=z_{s}-z_{m}$, and then

$$
\begin{gathered}
\dot{E}=\dot{w}_{m}+\dot{w}_{s}=M\left(z_{m}\right) w_{m}+M\left(z_{s}\right) w_{s}+u_{w}, \\
\dot{e}=\dot{z}_{s}-\dot{z}_{m}=N\left(w_{s}, z_{s}\right)-N\left(w_{m}, z_{m}\right)+u_{z} .
\end{gathered}
$$

Obviously, if $N(-w, z)=N(w, z)$, then $E=0, e=0$, are the equilibria of unforced nominal sum system (12) and error system (13), respectively. According to the nonlinear control theory, the controller designed can reach the coexistence of synchronization and antisynchronization of system (1) and (2).

\section{Illustrative Example}

In this section, we give two numerical examples to illustrate how to use the results we obtained in this paper to realize the synchronization and antisynchronization simultaneously and the coexistence of synchronization and antisynchronization, respectively. 
Example 14. Consider the following chaotic system [23]:

$$
\begin{aligned}
& \dot{x}_{1}=p x_{2}-\frac{2 p}{7} x_{1}^{3}+\frac{p}{7} x_{1}, \\
& \dot{x}_{2}=x_{1}-x_{2}+x_{3}, \\
& \dot{x}_{3}=-q x_{2},
\end{aligned}
$$

where $p=10$ and $q=100 / 7$, and system (14) is chaotic.

Obviously, system (14) satisfies Theorem 8; thus this system can realize the synchronization and antisynchronization simultaneously.

Let system (14) be the master system, and then the slave system is as follows:

$$
\begin{aligned}
& \dot{y}_{1}=p y_{2}-\frac{2 p}{7} y_{1}^{3}+\frac{p}{7} y_{1}+u_{1}, \\
& \dot{y}_{2}=y_{1}-y_{2}+y_{3}+u_{2}, \\
& \dot{y}_{3}=-q y_{2}+u_{3},
\end{aligned}
$$

where $u=\left(u_{1}, u_{2}, u_{3}\right)^{T}$ is the controller to be designed.

Let $e=y-x$, and if $e_{1}=0$, the following remainder error system

$$
\begin{aligned}
& \dot{e}_{2}=-e_{2}+e_{3}, \\
& \dot{e}_{3}=-q e_{2}
\end{aligned}
$$

is asymptotically stable. According to Lemma 7, the controller is designed as $u=\left(k_{1} e_{1}, 0,0\right)^{T}$, and $\dot{k}_{1}=-e_{1}^{2}$.

By the similar procedure, the controller $u=\left(k_{1} E_{1}, 0,0\right)^{T}$ can make master system (14) antisynchronizes slave system (15), where $\dot{k}_{1}=-E_{1}^{2}$.

Next, we give numerical simulations. The initial values of master system (14) and slave system (15) are $x(0)=$ $(1,-5,-4)^{T}, y(0)=(-2,3,1)^{T}$, and $k_{1}(0)=-1$. The simulation results are given in Figures 1 and 2, respectively.

Remark 15. From the results of numerical simulation, Figure 1 shows that system (14) and (15) reach synchronization, while Figure 2 shows that system (14) and (15) realize antisynchronization. Thus, the Chua chaotic system can realize the synchronization and antisynchronization simultaneously.

Example 16. Consider the following chaotic system [24]:

$$
\begin{aligned}
& \dot{x}_{1}=(25 \beta+10)\left(x_{2}-x_{1}\right), \\
& \dot{x}_{2}=(28-35 \beta) x_{1}+(29 \alpha-1) x_{2}-x_{1} x_{3}, \\
& \dot{x}_{3}=-\frac{1}{3}(8+\beta) x_{3}+x_{1} x_{2} .
\end{aligned}
$$

The above system is called unified chaotic system, where $\beta \epsilon$ $[0,1]$. If $\beta \in[0,0.8)$, the system is generalized Lorenz system; if $\beta \in(0.8,1]$, the system is generalized Chen system.

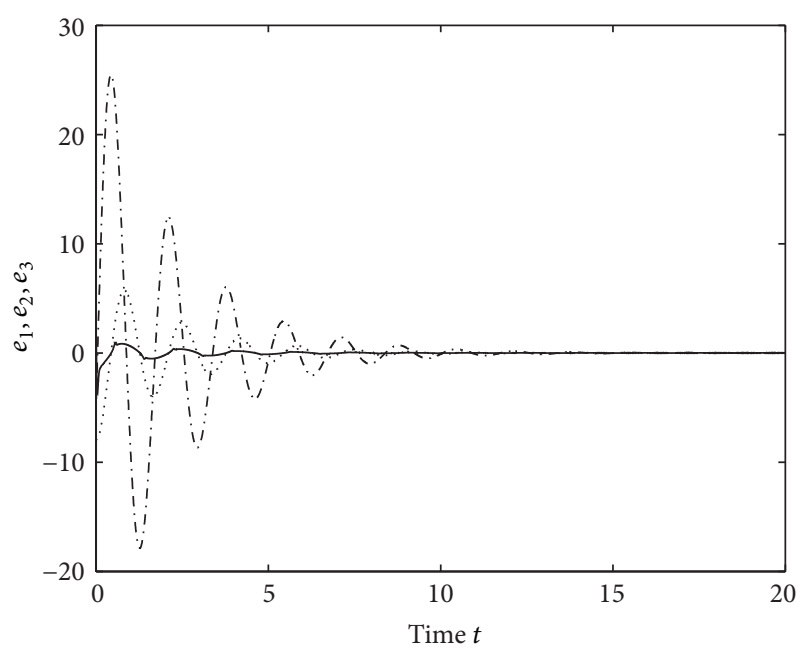

$$
\begin{array}{ll} 
& e_{1} \\
\ldots . & e_{2} \\
\ldots- & e_{3}
\end{array}
$$

FIGURE 1: The response of the error system.

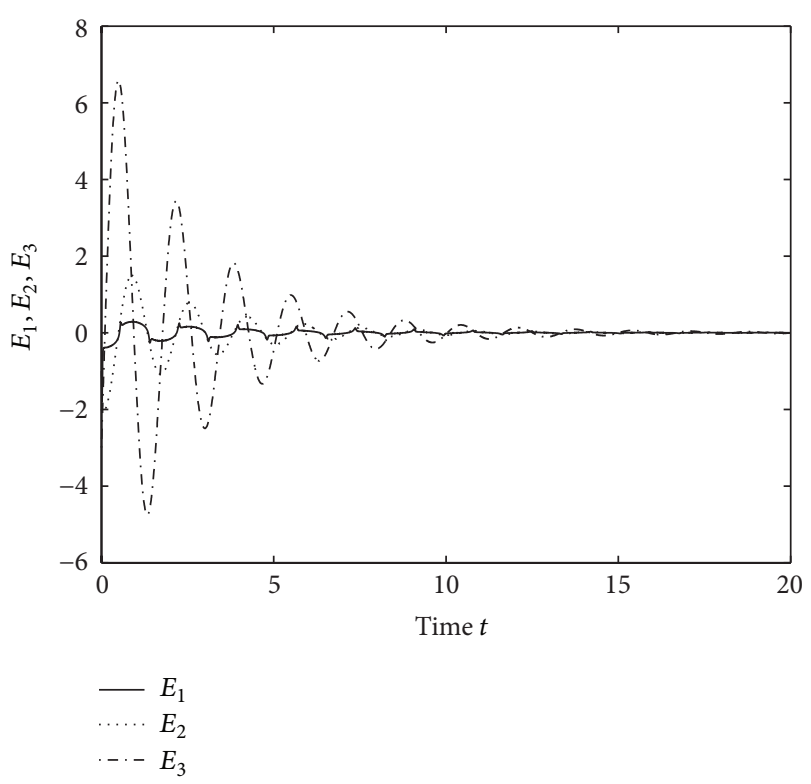

FIgURE 2: The response of the sum system.

Let system (17) be the master system, and then the slave system is described as

$$
\begin{aligned}
& \dot{y}_{1}=(25 \beta+10)\left(y_{2}-y_{1}\right)+u_{1}, \\
& \dot{y}_{2}=(28-35 \beta) y_{1}+(29 \beta-1) y_{2}-y_{1} y_{3}+u_{2}, \\
& \dot{y}_{3}=-\frac{1}{3}(8+\beta) y_{3}+y_{1} y_{2}+u_{3} .
\end{aligned}
$$


Let $e_{i}=y_{i}-\alpha_{i} x_{i}$, where $\left|\alpha_{i}\right|=1, i=1,2,3$. Then the unforced nominal error system is described as follows:

$$
\begin{aligned}
\dot{e}_{1}= & (25 \beta+10)\left(\left(y_{2}-\alpha_{1} x_{2}\right)-e_{1}\right), \\
\dot{e}_{2}= & (28-35 \beta) e_{1}+(29 \beta-1) e_{2}-e_{1} e_{3}-\alpha_{3} x_{3} e_{1} \\
& +\alpha_{1} x_{1} e_{3}+\left(\alpha_{1} \alpha_{3}-\alpha_{2}\right) x_{1} x_{3}, \\
\dot{e}_{3}= & e_{1} e_{2}+\alpha_{1} x_{2} e_{1}-\alpha_{2} x_{1} e_{2}+\left(\alpha_{1} \alpha_{2}-\alpha_{3}\right) x_{1} x_{2} \\
& -\frac{8+\beta}{3} e_{3} .
\end{aligned}
$$

According to Assumption 1,e $=0$ is the equilibrium of the above unforced nominal error system (19), and the following algebraical equations

$$
\begin{aligned}
\alpha_{2} & =\alpha_{1}, \\
\alpha_{1} \alpha_{3} & =\alpha_{2}, \\
\alpha_{1} \alpha_{2} & =\alpha_{3}
\end{aligned}
$$

should be satisfied.

By solving (20), we obtain two solutions: $\alpha_{i}=1, i=$ $1,2,3$, or $\alpha_{1}=\alpha_{2}=-1$ and $\alpha_{3}=1$. If $\alpha_{i}=1, i=$ $1,2,3$, which implies master system (17) and slave system (18) realize complete synchronization. If $\alpha_{1}=\alpha_{2}=-1$, $\alpha_{3}=1$, which implies that the first two variables of master system (17) antisynchronize the corresponding variables of slave system (18), while the third variable of master system (17) synchronizes the corresponding variables of slave system (18); that is, master system (17) and slave system (18) realize the coexistence of synchronization and antisynchronization.

Select $\alpha_{1}=\alpha_{2}=-1, \alpha_{3}=1$, and unforced nominal sum and error system (19) is given as

$$
\begin{aligned}
\dot{E}_{1}= & (25 \beta+10)\left(E_{2}-E_{1}\right) \\
\dot{E}_{2}= & (28-35 \beta) E_{1}+(29 \beta-1) E_{2}-E_{1} e_{3}-x_{3} E_{1} \\
& -x_{1} e_{3} \\
\dot{e}_{3}= & E_{1} E_{2}-x_{2} E_{1}+x_{1} E_{2}-\frac{8+\beta}{3} e_{3} .
\end{aligned}
$$

It is easy to obtain that if $E_{2}=0$, then remainder sum and error system (21)

$$
\begin{aligned}
& \dot{E}_{1}=-(25 \beta+10) E_{1}, \\
& \dot{e}_{3}=-x_{2} E_{1}-\frac{8+\beta}{3} e_{3}
\end{aligned}
$$

is asymptotically stable.
According to Lemma 7, forced sum and error system (21) is given as

$$
\begin{aligned}
\dot{E}_{1}= & (25 \beta+10)\left(E_{2}-E_{1}\right) \\
\dot{E}_{2}= & (28-35 \beta) E_{1}+(29 \beta-1) E_{2}-E_{1} e_{3}-x_{3} E_{1} \\
& -x_{1} e_{3}+k_{1} E_{2} \\
\dot{e}_{3}= & E_{1} E_{2}-x_{2} E_{1}+x_{1} E_{2}-\frac{8+\beta}{3} e_{3}
\end{aligned}
$$

that is, the controller is $u=\left(0, k_{1} E_{2}, 0\right)^{T}$ and $\dot{k}_{1}=-E_{2}^{2}$.

Next, for Example 16, we can obtain the same conclusion by using Theorem 13 .

Let $w_{m}=\left(x_{1}, x_{2}\right)^{T}, z_{m}=x_{3}$, and master chaotic system (17) is rewritten as

$$
\begin{aligned}
& \dot{w}_{m}=M\left(z_{m}\right) u_{m}=\left(\begin{array}{cc}
-(25 \alpha+10) & 0 \\
0 & 25 \alpha+10
\end{array}\right) w_{m}, \\
& \dot{z}_{m}=N\left(w_{m}, z_{m}\right)=-\frac{1}{3}(8+\alpha) z_{m}+h\left(w_{m}\right),
\end{aligned}
$$

where $h\left(w_{m}\right)=x_{1} x_{2}$. Slave chaotic system (18) is also rewritten as

$$
\begin{aligned}
\dot{w}_{s} & =M\left(z_{s}\right) u_{s} \\
& =\left(\begin{array}{cc}
-(25 \alpha+10) & 0 \\
0 & 25 \alpha+10
\end{array}\right) w_{s}+u_{w}, \\
\dot{z}_{s} & =N\left(w_{s}, z_{s}\right)=-\frac{1}{3}(8+\alpha) z_{s}+h\left(w_{s}\right)+u_{s},
\end{aligned}
$$

where $h\left(w_{s}\right)=y_{1} y_{2}$.

It is easy to see that master system (24) and slave system (25) satisfy the conditions of Theorem 13. Therefore, master system (24) and slave system (25) realize the coexistence of synchronization and antisynchronization.

Next, we give numerical simulations. The initial values of master system (17) and slave system (18) are $x(0)=$ $(-2,3,4)^{T}, y(0)=(4,-1,-2)^{T}, \beta=0.75$, and $k_{1}(0)=-1$. The simulation results are given in Figures 3 and 4, respectively.

Remark 17. From the results of numerical simulation, Figure 3 shows that sum and error system (23) is asymptotically stable, while Figure 4 shows that master system (17) and slave (18) realize coexistence of synchronization and antisynchronization.

\section{Conclusions}

In this paper, we have investigated the synchronization and antisynchronization for a class of chaotic systems. Firstly, a necessary and sufficient condition has been proposed, with which the master system can synchronize and antisynchronize the slave system simultaneously. Secondly, two methods have been obtained to realize coexistence of synchronization 


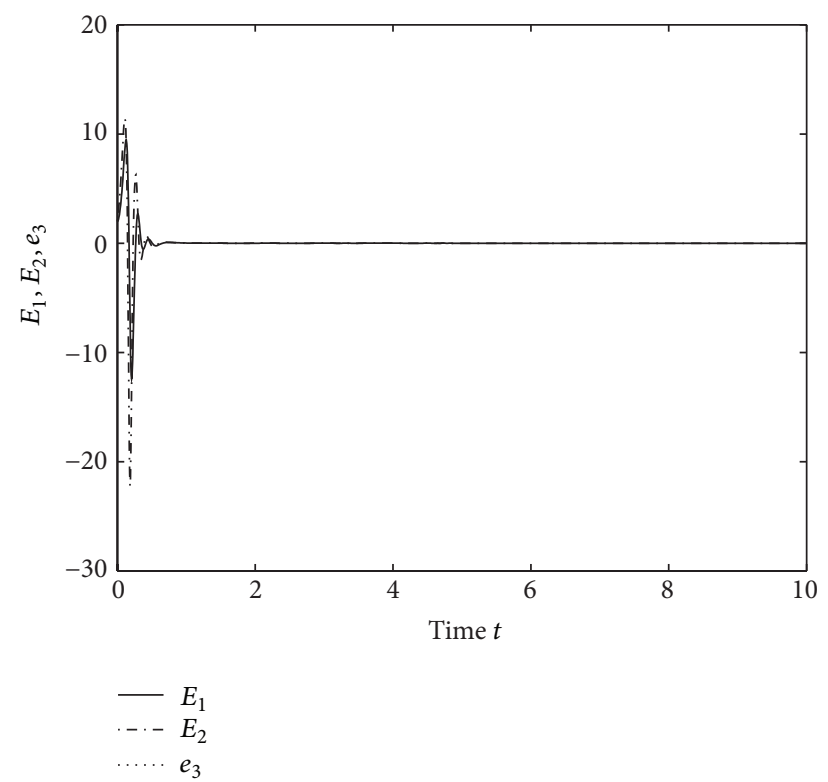

FIgURE 3: The response of sum and error system.
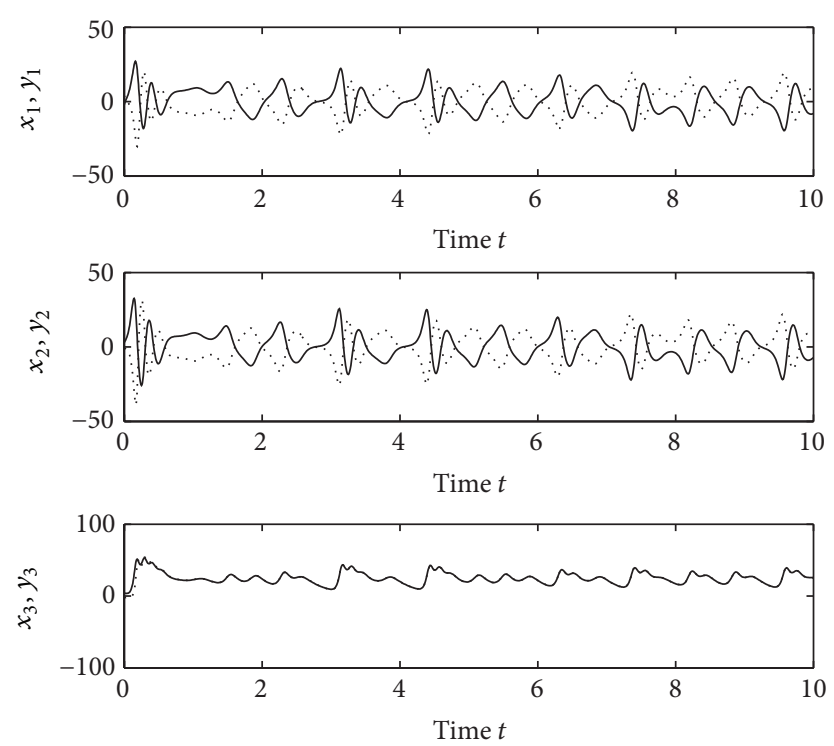

Figure 4: The response of master and slave system.

and antisynchronization for a class of chaotic systems, and the corresponding adaptive controllers have been also given. Two numerical examples with simulation have been used to verify the correctness and effectiveness of the obtained results.

\section{Conflict of Interests}

The authors declare that there is no conflict of interests regarding the publication of this paper.

\section{Acknowledgments}

This work was supported by National Natural Science Foundation of China [61304133, 61305130, and 61374074], China Postdoctoral Science Foundation funded project [2013M541915, 2013M541912, and 2014T70638], and the Scientific Research Foundation of Shandong Province Outstanding Young Scientist Award [BS2013SF023].

\section{References}

[1] E. N. Lorenz, "Deterministic nonperiodic flow," Journal of the Atmospheric Sciences, vol. 20, no. 2, pp. 130-141, 1963.

[2] O. E. Rössler, "An equation for continuous chaos," Physics Letters A, vol. 57, no. 5, pp. 397-398, 1976.

[3] J.-M. Grandmont, "On endogenous competitive business cycles," Econometrica, vol. 53, no. 5, pp. 995-1045, 1985.

[4] L. M. Pecora and T. L. Carroll, "Synchronization in chaotic systems," Physical Review Letters, vol. 64, no. 8, pp. 821-824, 1990.

[5] E. Ott, C. Grebogi, and J. A. Yorke, "Controlling chaos," Physical Review Letters, vol. 64, no. 11, pp. 1196-1199, 1990.

[6] D. Auerbach, C. Grebogi, E. Ott, and J. A. Yorke, "Controlling chaos in high-dimensional systems," Physical Review Letters, vol. 69 , no. 24, pp. 3479-3482, 1992.

[7] J. Sieber, O. E. Omel'Chenko, and M. Wolfrum, "Controlling unstable chaos: stabilizing chimera states by feedback," Physical Review Letters, vol. 112, no. 5, Article ID 054102, 2014.

[8] Z. Q. Zhang, S. Y. Xu, and B. Y. Zhang, "Asymptotic tracking control of uncertain nonlinear systems with unknown actuator nonlinearity," IEEE Transactions on Automatic Control, vol. 59, no. 5, pp. 1336-1341, 2014.

[9] Z. Q. Zhang, S. Y. Xu, and B. Y. Zhang, "Exact tracking control of nonlinear systems with time delays and dead-zone input," Automatica, vol. 52, pp. 272-276, 2015.

[10] Z. Q. Zhang and S. Y. Xu, "Observer design for uncertain nonlinear systems with unmodeled dynamics," Automatica, vol. 51, pp. 80-84, 2015.

[11] Z. Zhang and X.-J. Xie, "Asymptotic tracking control of uncertain nonlinear systems with unknown actuator nonlinearity and unknown gain signs," International Journal of Control, vol. 87, no. 11, pp. 2294-2311, 2014.

[12] D. Huang, "Simple adaptive-feedback controller for identical chaos synchronization," Physical Review E, vol. 71, no. 3, Article ID 037203, 2005.

[13] R. Guo, "A simple adaptive controller for chaos and hyperchaos synchronization," Physics Letters A, vol. 372, no. 34, pp. 55935597,2008

[14] S. Hammami, M. Benrejeb, M. Feki, and P. Borne, "Feedback control design for Rössler and Chen chaotic systems antisynchronization," Physics Letters A, vol. 374, no. 28, pp. 28352840, 2010.

[15] M. Mossa Al-Sawalha, M. S. Noorani, and M. Al-dlalah, "Adaptive anti-synchronization of chaotic systems with fully unknown parameters," Computers \& Mathematics with Applications, vol. 59, no. 10, pp. 3234-3244, 2010.

[16] D. L. Xu, W. L. Ong, and Z. G. Li, "Criteria for the occurrence of projective synchronization in chaotic systems of arbitrary 
dimension," Physics Letters A, vol. 305, no. 3-4, pp. 167-172, 2002.

[17] E. A. Barbashin, Introduction to the Theory of Stability, WoltersNoordhoff, Groningen, The Netherlands, 1970.

[18] L. Wu and S. Zhu, "Coexistence and switching of anticipating synchronization and lag synchronization in an optical system," Physics Letters, Section A: General, Atomic and Solid State Physics, vol. 315, no. 1-2, pp. 101-108, 2003.

[19] H.-T. Yau, "Synchronization and anti-synchronization coexist in two-degree-of-freedom dissipative gyroscope with nonlinear inputs," Nonlinear Analysis. Real World Applications, vol. 9, no. 5, pp. 2253-2261, 2008.

[20] Q. Zhang, J. Lü, and S. Chen, "Coexistence of anti-phase and complete synchronization in the generalized Lorenz system," Communications in Nonlinear Science and Numerical Simulation, vol. 15, no. 10, pp. 3067-3072, 2010.

[21] K. H. Wei, "The solution of a transcendental problem and its applications in simultaneous stabilization problems," IEEE Transactions on Automatic Control, vol. 37, no. 9, pp. 1305-1315, 1992.

[22] R.-W. Guo, "Simultaneous synchronization and anti-synchronization of two identical new 4D chaotic systems," Chinese Physics Letters, vol. 28, no. 4, Article ID 040205, 2011.

[23] M. T. Yassen, "Adaptive control and synchronization of a modified Chua's circuit system," Applied Mathematics and Computation, vol. 135, no. 1, pp. 113-128, 2003.

[24] Z. Q. Zhang, H. Shen, and J. L. Li, "Adaptive stabilization of uncertain unified chaotic systems with nonlinear input," Applied Mathematics and Computation, vol. 218, no. 8, pp. 42604267, 2011. 


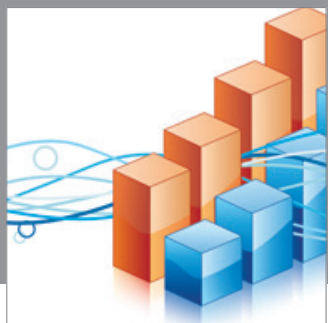

Advances in

Operations Research

mansans

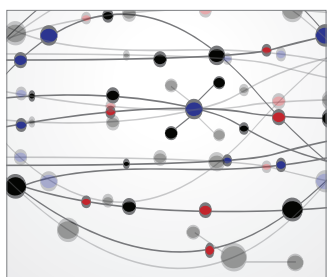

The Scientific World Journal
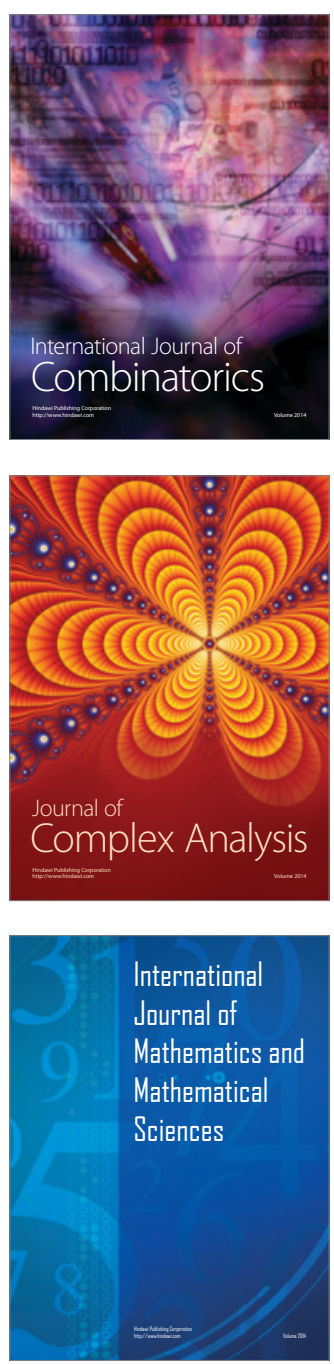
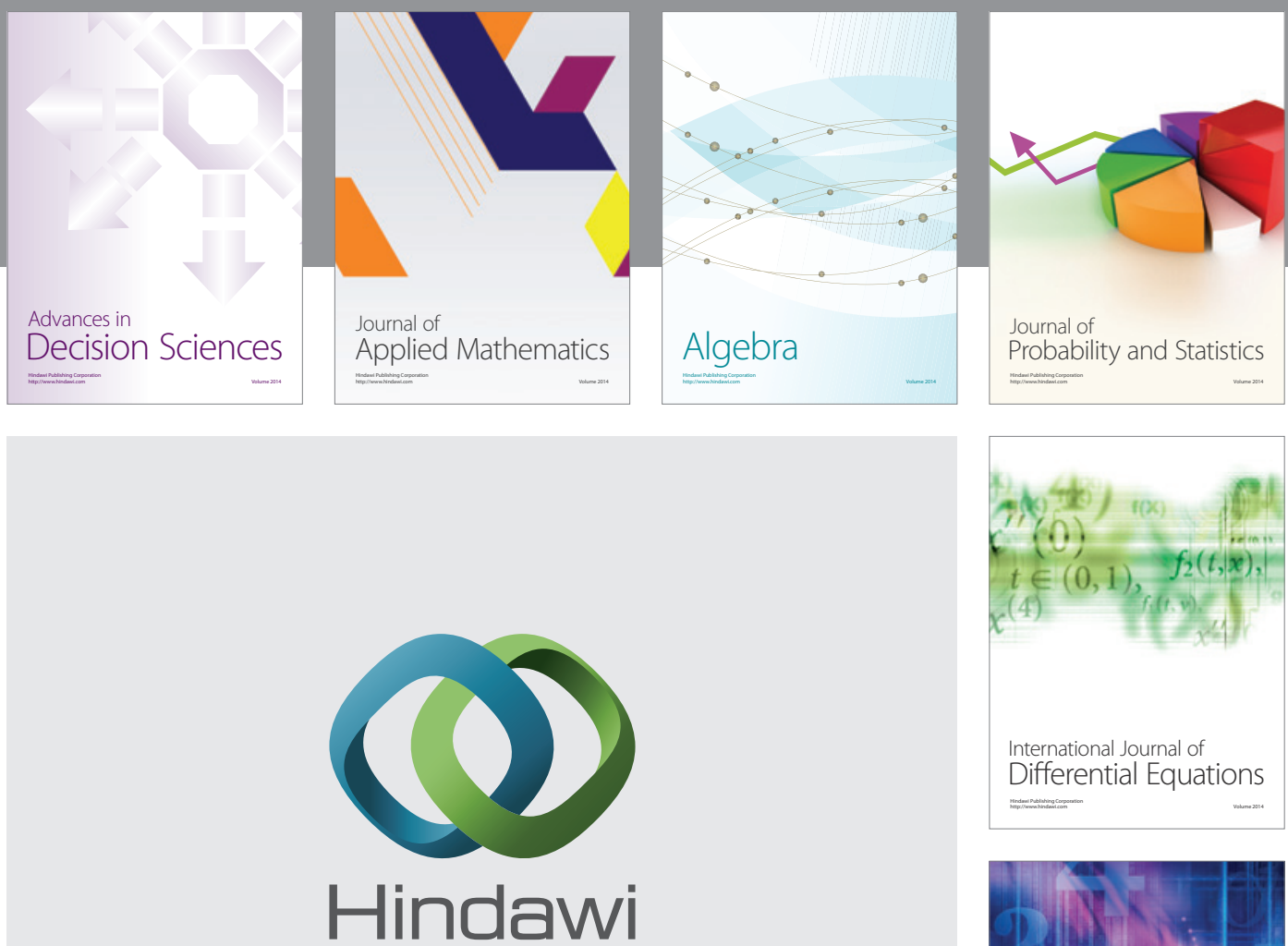

Submit your manuscripts at http://www.hindawi.com
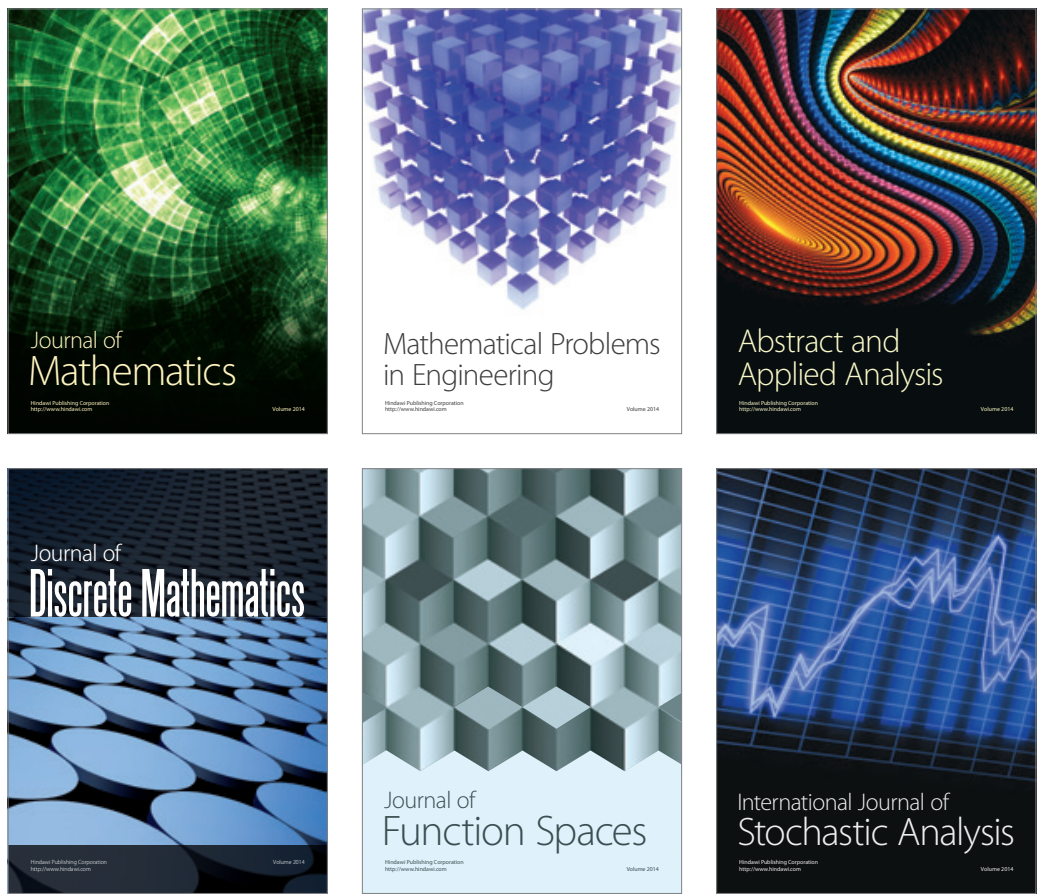

Journal of

Function Spaces

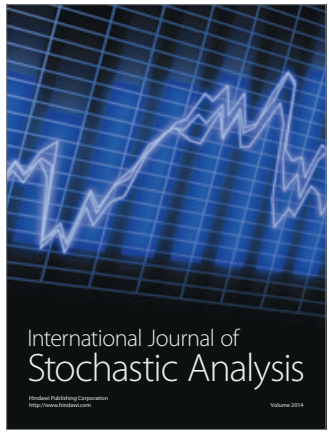

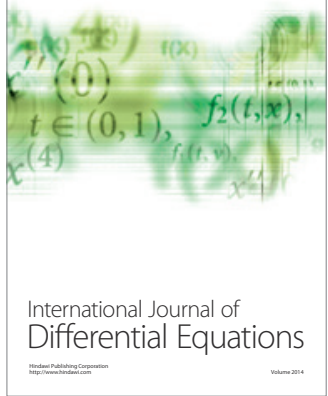
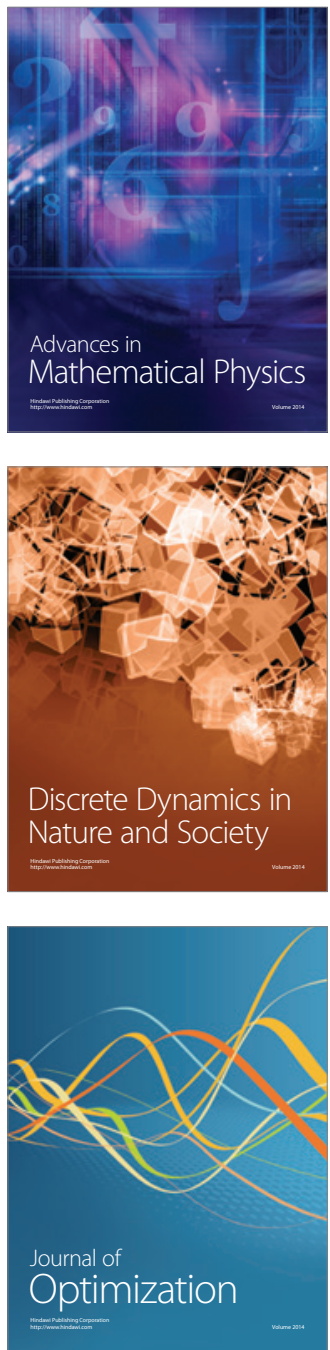